\title{
La creatividad en la enseñanza como factor de aproximación de la universidad a los desafíos sociales
}

\section{Creativity in teaching as a factor in bringing universities closer to social challenges}

\section{A criatividade no ensino como factor de aproximação da universidade aos desafios societais}

\author{
Ana Belén Fernández Souto \\ Profesora \\ (Universidad de Vigo) \\ https://orcid.org/0000-0003-2685-0604 \\ España \\ Sara Balonas \\ Centro de Estudos de Comunicação e Sociedade \\ (Universidade do Minho) \\ https://orcid.org/0000-0003-0885-1718 \\ Portugal
}

- Artículo invitado -

Fecha de recepción: 4 de junio de 2021

Fecha de publicación: 1 de julio de 2021

Para citar este artículo: Fernández Souto, A. y Balonas, S. (2021). La creatividad en la enseñanza como factor de aproximación de la universidad a los desafíos sociales, Icono 14, 19(2), 11-35. doi: 10.7195/ri14.v19i2.1754 


\section{Resumen}

El artículo propone la reflexión sobre la contribución de la creatividad como respuesta a los nuevos desafios globales a las que las sociedades están haciendo frente: la propuesta pretende repensar sobre las fórmulas que la enseñanza universitaria puede utilizar para estimular esta capacidad humana.

El trabajo se centra en la idea defendida por Wallas, que consideraba la creatividad como el resultado de un proceso evolutivo que permitía a los humanos adaptarse rápidamente a alteraciones de ambientes (1926). Teniendo en cuenta las alteraciones que la pandemia del Covid-19 ha provocado en las actividades sociales y económicas, parece necesario entender que los retos que afrontan las organizaciones deben ser asumidas también por las entidades académicas, por lo que las instituciones de enseñanza superior deben estar preparadas para formar profesionales aptos para tomar parte de la solución del problema. Por tanto, ¿qué competencias se exigen al nivel de formación?, ¿las universidades presentan una oferta de estudios orientada al ejercicio de la creatividad aplicada?, ¿qué características deben incluirse en la formación del profesional de comunicación dentro de su formación universitaria?

Con este fin, se propone una revisión del concepto de creatividad, con especial atención a la relevancia de la creatividad en el contexto científico y académico. A continuación, el artículo presenta el mapeo de la oferta formativa en este nivel, evaluando los planes de cursos de publicidad y relaciones públicas en Portugal y España (siguiendo el ranking de Shanghái), para entender si las instituciones de educación superior están preparadas o no para formar profesionales alineados con los requerimientos del nuevo panorama social tras la pandemia.

El trabajo concluye que la presencia de la creatividad en los planes de estudio de las principales universidades españolas y portuguesas es residual y prácticamente se limita a las especialidades vinculadas con la comunicación. Lejos de jugar un papel preponderante en la formación de los universitarios para dotarles de herramientas que les permitan desarrollarse profesionalmente en un entorno cambiante, el sistema de educación superior sigue viviendo de espaldas a las necesidades profesionales.

Palabras clave: Creatividad; Desafios sociales; Covid 19; Enseñanza universitaria; Relaciones Públicas y Publicidad 


\section{Abstract}

The article proposes a reflection on the contribution of creativity as a response to the new global challenges that societies are facing: the proposal aims to rethink the formulas that university education can use to stimulate this human capacity.

The work focuses on the idea defended by Wallas, who considered creativity as the result of an evolutionary process that allowed humans to adapt quickly to altered environments (1926). Given the disruptions that the Covid-19 pandemic has caused to social and economic activities, it seems necessary to understand that the challenges faced by organisations must also be taken on by academia, and higher education institutions must therefore be prepared to train professionals who can be part of the solution to the problem. So what skills are required at the level of education? Do universities offer courses geared towards the exercise of applied creativity? What characteristics should be included in the training of communication professionals as part of their university education?

To do this, a review of the concept of creativity is proposed, with particular attention to the relevance of creativity in the scientific and academic context. After that, the article presents the mapping of educational offerings at this level, evaluating the curricula of advertising and public relations courses in Portugal and Spain (following the ranking of Shanghai), to understand if higher education institutions are prepared or not to train professionals aligned with the requirements of the new social landscape after the pandemic.

The study concludes that the presence of creativity in the curricula of Spanish and Portuguese main universities is residual and is practically limited to the specialties linked to communication. Far from playing a major role in training university students to equip them with the tools to enable them to develop professionally in a changing environment, the higher education system continues to live behind its back on professional needs.

Keywords: Creativity; Social challenges; Covid 19; University teaching; Public Relations and Advertising 


\section{Resumo}

0 artigo propõe discutir o contributo da criatividade na procura de respostas para os novos desafios globais que as sociedades enfrentam, refletindo especificamente sobre a forma como o ensino universitário está preparado para tal. 0 trabalho parte da ideia fundadora de Wallas, que considerava a criatividade como o resultado do processo evolutivo, permitindo aos humanos adaptarem-se rapidamente a alterações de ambientes (1926). Tendo em consideração o profundo impacto da pandemia provocada pela Covid-19 ao nível sanitário, social, económico e ambiental, torna-se oportuno compreender os novos desafios que governos e organizações de todo o tipo enfrentam para depois discutir o papel das instituições de ensino na formação para a criatividade e para a inovação, como requisito para a formação de profissionais capacitados e participativos no desenho da sociedade pós pandemia. Que competências se exigem ao nível da formação? As universidades apresentam uma oferta formativa orientada para o exercício da criatividade aplicada?

Para isso, propõe-se uma revisitação do conceito de criatividade, com particular atenção para a relevância da criatividade no contexto científico e académico. Seguidamente, o artigo apresenta o mapeamento da oferta formativa a este nivel, avaliando os planos de cursos de Publicidade e Relações Públicas de Portugal e Espanha (seguindo o ranking de Shangai), para compreender se as instituições de ensino superior estão ou não preparadas para formar profissionais alinhados com as exigências do novo panorama societal pós pandemia.

0 estudo conclui que a presença da criatividade nos currículos das principais universidades espanholas e portuguesas é residual e praticamente limitada às especialidades ligadas à comunicação. Longe de desempenhar um papel preponderante na formação dos estudantes universitários para lhes proporcionar as ferramentas que lhes permitirão desenvolver-se profissionalmente num ambiente em mudança, 0 sistema de ensino superior continua a viver com as costas voltadas para as necessidades profissionais.

Palavras chave: Criatividade; Desafios societais; Covid-19; Ensino universitário; Relações Públicas e Publicidade 
La creatividad en la enseñanza como factor de aproximación de la universidad... | 15 MONOGRÁFICO

\section{Introducción}

Los nuevos retos sociales en general, y la pandemia provocada por el Covid-19 en particular, han desafiado la capacidad humana para generar nuevas soluciones en el ámbito de la salud, pero también a otros niveles. Ante un escenario sin precedentes a nivel planetario, 2020 corre el riesgo de ser considerado el año catalizador de nuevos enfoques a nivel social, económico, tecnológico y ambiental. En este orden de ideas, la creatividad puede ser una contribución nada desdeñable si seguimos la línea defendida por May (1975), cuando se refirió a la creatividad como el proceso de traer algo nuevo, que estaría oculto y apuntando a nuevos caminos.

Este artículo busca comprender la importancia de la creatividad en las sociedades actuales, asumiendo que el concepto es, en sí mismo, complejo, ya que la revisión bibliográfica lo evidencia al ofrecer una gran diversidad de enfoques. Necesitamos entender las distintas perspectivas como una forma de estabilización conceptual vinculada con la cuestión central de este trabajo: entender si la educación universitaria puede ser un inductor efectivo de la creatividad en las organizaciones, permitiendo la formación de profesionales para hacer frente a los grandes desafíos de un mundo VUCA (Volátil, Incierto, Complejo y Ambiguo).

Para ello, comenzamos caracterizando y distinguiendo las principales interpretaciones en torno al concepto de creatividad hasta encontrar la conceptualización dominante hoy en día, asumiendo desde el principio que los campos de aplicación de la creatividad son vastos, desde las artes, hasta la industria, las empresas e incluso los gobiernos. La discusión se llevará a cabo en torno a la triangulación Creatividad - Desafíos sociales en un marco post-pandemia - la enseñanza de la creatividad, con el fin de entender si las universidades son capaces de seguir los cambios de paradigma que se avecinan.

Esta triangulación se estudiará de forma cualitativa, partiendo de una investigación bibliográfica sobre la creatividad que permita delimitar el concepto dentro de la temática propuesta en este artículo, seguida de la caracterización de la crisis provocada por la pandemia, mediante la consulta y el estudio de datos e investigaciones actuales. A continuación, se realiza un análisis de contenido basado en 


\section{MONOGRÁFICO}

dos datos de las cinco universidades españolas y portuguesas mejor situadas en la última edición del Ranking de Shánghai, seleccionándose aquellas en las que se imparten grados o licenciaturas en comunicación y/o en publicidad y RRPP. En este sentido, la investigación también resulta descriptiva. La opción recae en el método inductivo, que parte de la observación de los fenómenos de creatividad, pandemia y enseñanza de la creatividad, correlacionándolos hacia una conclusión.

\section{Creatividad: formas de mirar}

Definir la creatividad es algo complejo ya que difiere según el contexto en el que se centre (Runco, 2006, cit. En David \& Morais, 2012). Históricamente, tal y como lo concebimos, es un término reciente, con poco más de un siglo. En la antigua Grecia, cualquier forma de arte, expresada en pintura o poesía, por ejemplo, no era creación, sino más bien "descubrimiento" (Balonas, 2019). En la cultura occidental, la noción de creatividad surge con el cristianismo, atribuido a la inspiración divina y no como un atributo del ser humano. La visión dominante fue proporcionada por la historia bíblica de la Creación dada por el Génesis (Boorstin, 1993). Con el Renacimiento, la creación se centra en los "grandes hombres" (Albert \& Runco, 1999), y ya no es una exclusiva de Dios. Los autores también sostienen que sólo con la Ilustración la creatividad se asocia con la imaginación.

Hay múltiples formas de analizar el fenómeno de la creatividad. A menudo se asocia con la obra de Leonardo da Vinci, Rodin o Frida Kahlo, pero también con el teléfono de Alexander Graham Bell o con la lámpara Edison. En estos casos, la creatividad se atribuye a un individuo y se asocia con algo tangible, como una pintura, una escultura, un soneto o una invención. El producto es posible verlo y estudiarlo (De Masi, 2002). Sin embargo, De Masi llama la atención sobre la creatividad como acto colectivo y su influencia en las sociedades: “la mayoría de los productos estéticos y científicos -películas, publicidad, diseño, planes urbanos, descubrimientos científicos y drogas biológicas, escenarios económicos- son el resultado de la creación colectiva" (2002).

Así, abordamos conceptos de creatividad orientados a respuestas concretas a retos sociales, primero muy ligados a "invenciones" individuales $\mathrm{y}$, posteriormente, a grupos que se caracterizan por la creatividad colectiva. 


\section{El valor de la creatividad en el mundo contem- poráneo}

En las sociedades contemporáneas, la creciente atención a los usos de la creatividad conduce a la identificación de subáreas, como las identificadas por Florida (2013): creatividad tecnológica (invención), creatividad económica (emprendimiento) y creatividad cultural/artística. Para el autor, estas dimensiones están interrelacionadas, compartiendo un proceso de razonamiento común y refuerzo entre sí. La economía creativa, por otro lado, es el resultado de las interacciones entre la tecnología, el arte y los negocios (Hollanders \& Cruysen, 2009).

La creatividad es muy valorada en la actualidad. Para Damásio (1994), nuestras estrategias de razonamiento son defectuosas, argumentando que, aunque las estrategias de razonamiento estén perfectamente afinadas, no están en línea con la incertidumbre y complejidad de los problemas personales y sociales, una idea que nos parece muy actual si la relacionamos con la crisis sanitaria de 2020 provocada por la pandemia y la ausencia de planes o estrategias oportunas para hacerle frente.

Por lo tanto, se puede admitir que el siglo XXI ve una creciente revalorización de la creatividad (Pink, 2005) en una era conceptual en la que necesitamos alimentar y fomentar el lado derecho del cerebro (creatividad y emoción), más que el lado izquierdo (del pensamiento lógico y analítico).

Frente a la idea de que la creatividad es una capacidad reservada sólo para unos pocos y reforzando, en cierto sentido, el valor de la creatividad colectiva, Kaufman \& Beghetto (2009) explican los diversos tipos de creatividad que se pueden desarrollar. Como explican en su modelo 4Cs, hay 4 tipos de creatividad: 1) la pequeña C - la creatividad del día a día; 2 ) la gran C-creatividad reservada para las mentes brillantes; 3) el mini C - un proceso de aprendizaje; y 4) pro C - la progresión que representa el nivel profesional. Claramente, Florida (2013) apoya su argumento en este último tipo de creatividad -la pro C- cuando evoca la creatividad tecnológica, muy presente en la aceleración digital de las organizaciones en el contexto de la pandemia causada por el Covid 19. Pero también a la hora de defender la creativi- 


\section{MONOGRÁFICO}

dad económica, que no es más que la búsqueda de nuevos modelos de negocio y/o adecuación de los existentes como forma de supervivencia.

Así, avanzamos hacia la creatividad orientada, es decir, con el propósito de resolver problemas, acercándonos a la dimensión de pro $\mathrm{C}$ mencionada anteriormente y teniendo en cuenta que la creatividad ocurre en la exigente duplicidad de la originalidad con la eficacia (Morais, 2011, cit. en Balonas, 2019). Esta perspectiva transporta el concepto de creatividad al universo de las organizaciones $\mathrm{y}$, sobre todo, al imperativo de la innovación en un contexto de gran competitividad: "las organizaciones tienen una necesidad constante de mejorar su ventaja competitiva y responder más rápidamente a los mercados cambiantes, reduciendo costes, mejorando la calidad, volviéndose orientadas al cliente, aumentando la productividad e innovando" (Stenfors \& Tanner, 2006).

Hoy en día, el concepto de creatividad es multidimensional. Se aplica a las artes y la literatura, las áreas científicas, los medios de comunicación, el mundo de los negocios, las industrias e incluso los gobiernos... pero pocas veces se relaciona con la capacidad de generar respuestas (Balonas, 2019). La creatividad es, en este orden de ideas, un factor de innovación que produce nuevas soluciones e involucra a las más diversas entidades, desde el Estado, hasta las empresas y/o la sociedad civil, pero también a las instituciones educativas.

\section{De la creatividad científica a los científicos em- prendedores}

Una mirada a la realidad de las universidades nos permite identificar tendencias de lo que se puede entender como creatividad científica al servicio de las empresas y la sociedad. Esta es una predisposición fácilmente reconocible, ya que cada vez se fomenta más la transferencia de conocimiento, a través de consorcios y proyectos que estimulan la creación de equipos interdisciplinarios y académicos, emprendedores y gestores públicos. Es decir, crear equipos colaborativos con universidades, centros de investigación o de conocimiento, think tanks, entre otros modelos. 
“La idea de que la ciencia es un esfuerzo creativo es innegable. Las ideas científicas son creaciones de la mente. La invención, por supuesto, de conceptos y teorías, la mayoría de las veces, requiere saltos imaginativos extraordinarios, pero también es cierto que esto aplica incluso en el trabajo científico cotidiano, como el descubrimiento y la resolución de problemas, la formación de hipótesis y el modelado, el pensamiento nativo / creativo, son fundamentales..." (Hadzigeorgiou et al., 2012). Por lo tanto, se puede argumentar que la creatividad siempre ha existido en la academia, relacionada con los avances en el conocimiento científico, y es razonable identificar la "pequeña creatividad C" de cada día con la "ciencia normal" y la "gran creatividad C" con la "ciencia revolucionaria" (Hadzigeorgiou et al., 2012).

Mirando los últimos 30 años, se entiende que las universidades han desarrollado un papel importante como agentes del desarrollo económico y social, destacando dos tendencias crecientes: por un lado, la "normalización" del modelo de emprendimiento académico -que fomenta la explotación comercial de la investigación, por otro, la aparición de científicos emprendedores- que concilian el trabajo investigadore con el empresarial.

Si el campo científico parece seguir los significados dominantes dados a la creatividad en el siglo XXI, es importante entender si la enseñanza de la creatividad acompaña a los signos problemáticos de los tiempos.

\section{Crisis pandémica de 2020, desafíos y creatividad}

En respuesta a la crisis financiera de 2008, Florida (2013) argumentó que las empresas creativas y basadas en el conocimiento - particularmente en el campo de la alta tecnología - se estaban consolidando en las zonas urbanas, generando un nuevo modelo de crecimiento económico. Para el académico, estaríamos en la era de valorar a las personas $\mathrm{y}$, por lo tanto, la importancia de las universidades en este proceso: "solíamos pensar en nuestras instituciones ancla como nuestras grandes fábricas. (...) Pero en las ciudades maduras, universidades, colegios, centros de conocimiento de ideas, estas son instituciones ancla de talento, no sólo porque 
son generadores económicos directos, sino porque provocan un flujo de talento" (Florida, p. 3, 2013).

En 2020, la Organización Mundial de la Salud (OMS, 2020a) denominó a la enfermedad causada por un síndrome respiratorio agudo grave de "coronavirus (SRACoV-2) covid-19" y del 11 de febrero al 11 de marzo, declaró oficialmente la transición a un estado de pandemia (0MS, 2020b). El mundo entero se vio inmerso en desafíos sin precedentes en términos de salud pública, pero también en lo referido al nivel social, ambiental, empresarial, económico, científico y académico.

A efectos de este artículo, es importante entender el mundo post-Covid desde la perspectiva de la formación de los ciudadanos y las características más valoradas en los profesionales del futuro, como actores en el proceso de superación, en dos niveles: 1) ¿qué competencias se valorarán? 2) ¿Cuál es el papel de la enseñanza y la enseñanza de la creatividad, en particular, como respuesta a este nuevo mundo?

Según Florida (2013), el mercado laboral se está bifurcando, con la creciente apreciación de los trabajos altamente calificados vinculados al conocimiento, el tecnicismo y la infotecnología, así como los trabajos artísticos y creativos. Al mismo tiempo, el entorno manufacturero tiende a desaparecer y los empleos de mediana calificación serán aniquilados o al menos estabilizados. Un estudio del Instituto Mckinsey sobre el futuro del trabajo post-Covid-19 (Lund et al., 2021) apunta a la apreciación salarial de los trabajadores con más habilidades sociales y emocionales, así como habilidades tecnológicas.

Por su parte, Gardner (2010) ha defendido el papel crucial de la creatividad como una de las cinco habilidades cognitivas que los líderes del futuro deben tratar de cultivar.

Podemos concluir que el mercado valora a los profesionales capacitados para ejercer la creatividad orientada a resolver retos complejos. En este contexto, ¿están las universidades alineadas con las expectativas del mundo contemporáneo? 


\section{El impacto de la pandemia en las universidades}

En primer lugar, es importante entender que la capacidad de adaptación a nuevos entornos (con creatividad) debe comenzar dentro de las propias universidades y en sus procesos docentes, en función de los cambios que el propio sistema educativo ha experimentado durante el año 2020 y 2021. Con la pandemia, la mayoría de los gobiernos de todo el mundo cerraron temporalmente las instituciones educativas en un esfuerzo por contener la propagación del COVID-19 (0ssiannilsson, 2020), en consecuencia, la educación se volvió en líneal y remota. La Organización de las Naciones Unidas para la Educación, la Ciencia y la Cultura (UNESCO) estimó que la pandemia de COVID-19 trajo una interrupción educativa sin precedentes con 1,2 mil millones de estudiantes y jóvenes de todo el planeta afectados por el cierre de escuelas (2020). Sin embargo, también se abrió la discusión sobre nuevos paradigmas en el proceso de enseñanzaaprendizaje, en una reinvención que necesariamente convoca a la creatividad. Dadas las experiencias educativas que se han producido -por el uso de nuevos enfoques metodológicos y por la banalización en el uso de las nuevas tecnologías-, es lógico que la educación universitaria se enfrente a un momento de transformación, consecuencia de todas las potencialidades que estos duros aprendizajes han traído consigo.

\section{La enseñanza universitaria de la creatividad en España y Portugal}

El sistema universitario, tanto en España como en Portugal, incluye la enseñanza de los aspectos creativos en diversos títulos, tanto en grados como en cursos de especialización. Los contenidos creativos suelen estar presentes en los ámbitos de ciencias sociales y humanidades, así encontramos, por ejemplo, el Grado en literatura y escritura creativa (Universidad de Navarra), Máster en psicocreatividad (Universidad Autónoma de Barcelona), Máster en Economía creativa (Universidad Rey Juan Carlos), Experto en Creatividad aplicada (Universidad Autónoma de Madrid), Experto en neuroeducación y creatividad (Universidad de Burgos)... La oferta es más abundante en los campos de la comunicación, educación, psicología, bellas artes y literatura, pero prácticamente nula en otras especialidades más técnicas, caso de las arquitecturas o ingenierías, donde bien podríamos pensar que la creatividad y el ingenio deberían estar muy presentes. 
Algunas universidades ofertan materias de carácter optativo sobre contenidos relacionados con la creatividad y lo hacen intentando formar parte de una base transversal, caso de la Universidad Complutense de Madrid con "Técnicas de estimulación creativa" o "Coaching y creatividad". Sin embargo, estos ejemplos son bastante escasos.

En el ámbito más ligado a la comunicación, la creatividad suele ser objeto de estudio dentro del mundo publicitario, pero no como materia amplia aplicable a otras disciplinas. En la práctica totalidad de las licenciaturas y grados ofertados en comunicación, las materias que directamente tratan los aspectos creativos son aquellas relacionadas con la publicidad. En el caso de los posgrados y doctorados, existen casos de oferta genérica que utilizan la expresión de "comunicación social" o "comunicación" (Castillo Esparcia, A., 2013) pero también en estos casos los contenidos directamente relacionados con la creatividad son poco visibles (Cuenca-Fontbona J., Compte-Pujol, M., Matilla K.,Hernández-Martínez, S., 2017).

Según la plataforma universia.net (https://www.universia.net/es/home.html ), en España se imparten 110 grados en comunicación (incluyendo las ramas tradicionales de periodismo, comunicación audiovisual y publicidad y Relaciones Públicas; pero también nuevas modalidades como protocolo y organización de eventos), 113 máster en comunicación, 120 cursos de posgrado y 33 de doctorado. El caso portugués, cuenta con un menor número total, pero guarda una proporción similar: 39 cursos de grado en comunicación, 31 programas de mestrado, 10 de postgrado y 12 de doctorado).

Si nos detenemos a analizar el plan de estudios de estas modalidades vemos que las materias que trabajan el ámbito creativo son escasísimas y, generalmente, vinculadas en exclusiva al ámbito del pensamiento creativo en la publicidad. Según Peyró, Serrano y Lorenzo (2010), se entiende que estas asignaturas de Creatividad publicitaria están orientadas hacia el desarrollo y potenciación de las capacidades intelectuales y artísticas que el alumno necesitará para la realización profesional de campañas de comunicación eficaces. Indica también que la trascendencia de esta asignatura dentro del plan de estudios de la titulación 
estriba en la capacidad que aporta la creatividad a la hora de generar ideas que permitan diferenciar, personalizar y aportar valores extrínsecos a un producto, marca, empresa u organización.

Veámoslo:

Según el ranking de Shanghái de 2020 (https://www.shanghairanking.com/), las cinco mejores universidades españolas son, por este orden, la Universitat de Barcelona, la Universidad Complutense de Madrid, Universidad de Granada, Universidad de Valencia y la Universitat Autònoma de Barcelona. En el caso de Portugal, el listado lo encabezan la Universidade de Lisboa, Universidade de Porto, Universidade do Minho, Universidade de Aveiro y Universidade de Coimbra. Las realidades de oferta universitaria de uno y otro país son similares pero diferentes. En el caso español, los grados suelen ofertar especialidades de comunicación independientes: Grado en Periodismo, Grado en Comunicación Audiovisual y Grado en Publicidad y RRPP, a mayores de ofertas emergentes vinculadas con la comunicación que responden a combinaciones de distintas especialidades. En el caso de Portugal, lo habitual es que la oferta de licenciatura sea genérica, en Comunicación.

En cualquier caso, si contemplamos el plan docente de los grados más próximos a la Publicidad y Relaciones Públicas vemos que:

\begin{tabular}{|l|l|l|}
\hline \multicolumn{1}{|c|}{ Universidad } & \multicolumn{1}{|c|}{ link } & $\begin{array}{l}\mathbf{N}^{0} \text { de materias } \\
\text { de creatividad }\end{array}$ \\
\hline $\begin{array}{l}\text { UB } \\
\text { Grado en } \\
\text { Publicidad, RRPP } \\
\text { y Márketing }\end{array}$ & $\begin{array}{l}\text { https://www.esrp.net/grau-en-publicitat-rela- } \\
\text { cions-publiques-i-marqueting/ }\end{array}$ & $\begin{array}{l}1 \\
\text {-Taller de } \\
\text { creatividad }\end{array}$ \\
\hline $\begin{array}{l}\text { UCM } \\
\text { Grado en Publicidad } \\
\text { y RRPP }\end{array}$ & $\underline{\text { https://www.ucm.es/gradopublicidad/estructu- }}$ & $\begin{array}{l}1 \\
\text {-El proceso de crea- } \\
\text { ción publicitaria }\end{array}$ \\
\hline $\begin{array}{l}\text { UGR } \\
\text { Grado en } \\
\text { Comunicación } \\
\text { Audiovisual }\end{array}$ & $\begin{array}{l}\text { https://grados.ugr.es/audiovisual/pages/infoa- } \\
\text { cademica/estudios }\end{array}$ & 0 \\
\hline
\end{tabular}




\begin{tabular}{|l|l|l|}
\hline \multicolumn{1}{|c|}{ Universidad } & \multicolumn{1}{|c|}{ link } & $\begin{array}{l}\mathbf{N}^{\mathbf{0}} \text { de materias } \\
\text { de creatividad }\end{array}$ \\
\hline $\begin{array}{l}\text { UV } \\
\text { Grado en } \\
\text { Audiovisual }\end{array}$ & $\begin{array}{l}\text { https://www.uv.es/uvweb/grado-comunica- } \\
\text { cion-audiovisual/es/grado-comunicacion-audio- } \\
\text { visual-1285938936332.html }\end{array}$ & 0 \\
\hline $\begin{array}{l}\text { UAB } \\
\text { Grado en Publicidad } \\
\text { y RRPP }\end{array}$ & https://www.uab.cat/Document/417/685/972P & $\begin{array}{l}\text {-Procesos y } \\
\text { técnicas creativas } \\
\text {-Creatividad en } \\
\text { publicidad y RRPP } \\
\text {-0P. Creatividad } \\
\text { gráfica }\end{array}$ \\
\hline
\end{tabular}

Tabla 1: Universidades líderes del ranking de Shanghái 2020 en España. Materias específicas de creatividad en los planes de estudio de grados de Publicidad y Relaciones Públicas.

Fuente: Elaboración propia a partir de https://www.universityrankings.ch/ results? ranking $=$ Shanghai\&region $=$ World\&year $=2020 \& q=$ Spain

Del plan de estudios de los grados de Publicidad y Relaciones Públicas que actualmente imparten las universidades españolas mejor posicionadas en el Ranking de Shanghái, vemos que la oferta de materias relacionadas con la creatividad difiere de unas a otras. Si bien todas cuentan con una asignatura que específicamente incluye "creatividad" en su nomenclatura, solamente la Universidad Autónoma de Barcelona lleva estos contenidos al ámbito publicitario y al campo de las relaciones públicas $\mathrm{y}$, a mayores, oferta materias optativas para sus alumnos. Todos los planes de estudio incluyen un seminario sobre la creatividad y lo aplican directamente a la Publicidad, pero no es habitual que lo lleven a los otros ámbitos profesionales $\mathrm{y}$, cuando lo hacen, los contenidos creativos son asumidos desde asignaturas vinculadas a la estrategia de comunicación y no directamente a la creatividad.

Aquellas universidades que no ofertan grado en Publicidad y RRPP pero sí en comunicación audiovisual, no integran en su plan de estudios materias específicas de creatividad. 


\begin{tabular}{|c|c|c|}
\hline Universidad & link & $\begin{array}{l}N^{0} \text { de materias } \\
\text { de creatividad }\end{array}$ \\
\hline $\begin{array}{l}\text { ULISBOA } \\
\text { Lic. Comunicaçao }\end{array}$ & $\begin{array}{l}\text { https://www.iscsp.ulisboa.pt/pt/cursos/oferta- } \\
\text { graduada/licenciaturas/ciencias-da-comunica- } \\
\text { cao/plano-de-estudos }\end{array}$ & $\begin{array}{l}0 \\
\text { Sin materias específicas } \\
\text { en creatividad }\end{array}$ \\
\hline $\begin{array}{l}\text { UPORT0 } \\
\text { Lic. Comunicaçao }\end{array}$ & $\begin{array}{l}\text { https://sigarra.up.pt/flup/pt/cur geral.cur } \\
\text { view?pv_curso_id=455 }\end{array}$ & $\begin{array}{l}0 \\
\text { Sin materias específicas } \\
\text { en creatividad }\end{array}$ \\
\hline $\begin{array}{l}\text { UMINHO } \\
\text { Lic. Publicidade } \\
\text { e RRPP }\end{array}$ & $\begin{array}{l}\text { https://www.ics.uminho.pt/pt/Estudar/Licen- } \\
\text { ciaturas/Ciencias-da-Comunicacao/Paginas/ } \\
\text { Plano-de-Estudos.aspx }\end{array}$ & \begin{tabular}{|l|}
4 \\
Criatividade em Publici- \\
dade I y II, Criatividade \\
em RRPP I y II \\
\end{tabular} \\
\hline $\begin{array}{l}\text { UCOIMBRA } \\
\text { Lic Jornalismo e } \\
\text { Communicaçao }\end{array}$ & $\begin{array}{l}\text { https://apps.uc.pt/courses/PT/ } \\
\text { programme/5341/2021-2022?id branch=15521 }\end{array}$ & 0 \\
\hline $\begin{array}{l}\text { UNOVALISBOA } \\
\text { Lic en CC da } \\
\text { Comunicaçao }\end{array}$ & $\begin{array}{l}\text { https://guia.unl.pt/pt/2020/fcsh/ } \\
\text { program/9023\#structure }\end{array}$ & 0 \\
\hline
\end{tabular}

Tabla 2: Universidades líderes del ranking de Shanghái 2020 en Portugal. Materias específicas de creatividad en los planes de estudio de grados de comunicación.

Fuente: Elaboración propia a partir de https://www.universityrankings.ch/ results? ranking=Shanghai\&region=World\&year $=2020 \& q=$ Portugal

Como indicábamos y frente al panorama español, en estas universidades se imparten licenciaturas genéricas en comunicación y solamente en el caso de la Universidade de Minho identificamos claramente materias en las que se trabajen los contenidos creativos, desarrollados en dos cursos para las especialidades de publicidad y RRPP. En las otras universidades, la creatividad aparece integrada tangencialmente en una materia de publicidad y RRPP (caso de Universidade de Lisboa) y en el resto de casos, no se llega a identificar nominativamente en el plan de estudios.

Según estos datos, podríamos interpretar que el peso que la creatividad tiene en el sistema de enseñanza universitario de la Península Ibérica es escaso y casi residual, ya que lejos de estar presentes en la mayor parte de la oferta universitaria, la creatividad queda prácticamente circunscrita a la formación en Publicidad y Relaciones Públicas y, dentro de ella, casi en exclusiva al campo publicitario. 
Además, esta vinculación entre publicidad y creatividad aparece, muchas veces, desdibujada en materias cuya nomenclatura no incluye la palabra "Creatividad", caso de materias como "Redacción publicitaria" y "Dirección de arte", tal y como indicaba Rodríguez (2009).

Si nos centramos en las titulaciones que imparten Publicidad y Relaciones Públicas, debemos entender que los contenidos y materias deben atender en igualdad a ambas disciplinas, la publicidad y las Relaciones Públicas; sin embargo, los planes de estudio no suelen profundizar en el peso de la creatividad en el campo de las Relaciones Públicas y cuando incluyen conceptos creativos lo hacen a través de materias que versan sobre estrategia. Esta misma situación se repite en las otras especialidades de comunicación, caso de los tradicionales Periodismo y Comunicación Audiovisual donde las materias de creatividad brillan por su ausencia y donde su desarrollo laboral requiere, de forma necesaria, de conceptos y estrategias creativas. Los nuevos grados vinculados con la comunicación tampoco incluyen de forma directa los contenidos creativos, así lo vemos en los grados de protocolo y organización de eventos. Tampoco mejora la tendencia y analizamos los dobles y triples grados (Beriain A \& Gascón JFF, 2013) que mezclan disciplinas de comunicación con otras conceptualmente próximas, caso del marketing. En estos casos, la creatividad aparece vinculada a los contenidos publicitarios y brilla por su ausencia en las demás especialidades.

\section{Creatividad, universidad y pandemia}

Si algo hemos aprendido con la pandemia del Covid-19 es que debemos aumentar nuestra capacidad de adaptación ante situaciones como la crisis sanitaria que estamos viviendo. Si la creatividad es una interacción entre la persona y el entorno, donde el individuo debe adaptarse para sobrevivir, parece necesario que los estudiantes adquieran y profundicen en los recursos creativos, ya que serán la base que les permita desarrollar su vida laboral de forma eficaz y eficiente en un entorno determinado.

Si esta necesidad parece evidente para todas las profesiones, en el caso de los especialistas en comunicación se hace especialmente patente, no sólo en el ámbito 
La creatividad en la enseñanza como factor de aproximación de la universidad... | 27 MONOGRÁFICO

publicitario, sino de forma singular en el caso de las relaciones públicas y la comunicación organizacional.

La comunicación de crisis responde a la necesidad de adaptarse al cambio. Adaptación y creatividad parecen las claves para salir airoso de una crisis, sea cual sea, ya que nos aportarán herramientas que nos ayuden a conseguir los objetivos planteados y adaptarnos al cambio que impone la crisis.

La propia Pandemia del COVID-19 nos ha obligado a cambiar incluso modos de vida, desde la suspensión de la actividad pública en prácticamente todo el mundo, al teletrabajo y la teledocencia. En ese sentido, el mundo empresarial que se conocía hasta ahora no va a regresar y las organizaciones deben integrarse en un nuevo modelo económico, más rápido y prácticamente virtual. El teletrabajo se convirtió en algo habitual durante la pandemia gracias a la Cloud Computing, permitiendo a los colaboradores acceder a datos y sistemas de sus organizaciones para realizar las tareas. Los servicios en la nube están siendo cada vez más utilizados y parece que, tras el coronavirus, esta tendencia continuará. Muchas empresas pasarán a tener gran parte de sus estructuras en la nube para favorecer aún más la home office (Fontes, 2020). Un estudio del Kaizen Institute concluye que el 70\% de las empresas portuguesas pretenden adoptar un sistema mixto de trabajo tras la pandemia (Silva, 2020) y este nuevo sistema implica que los perfiles profesionales deben adaptarse.

Por tanto, necesitaremos profesionales capaces de adaptarse a los rápidos avances de la tecnología y de acompañar a sus organizaciones en los procesos de transformación hacia un escenario globalizado, tecnificado y volátil, recordando que, a excepción de China, todo el mundo opera con las mismas herramientas (Google, Facebook, Amazon, Salesforce...) es decir, todos tienen la misma manera de trabajar y gestionar los negocios.

Así, la inesperada pandemia del Covid ha modificado el día a día de las organizaciones más allá de los propios sistemas de producción. Los responsables de la comunicación se han visto obligados a adaptarse al entorno impuesto por la situación sanitaria y han tenido que planificar acciones en un escenario de crisis 


\section{MONOGRÁFICO}

total, partiendo de las herramientas tradicionalmente utilizadas e implementando técnicas, tácticas y estrategias llenas de creatividad para alcanzar los objetivos comunicativos.

Así hemos visto cómo en un primer momento de parón institucional, empresarial y personal, se suspendió toda la actividad social y pública, lo que hizo que todo mensaje comunicativo llegase a sus distintos públicos desde "el más allá", a través del teletrabajo, la tele docencia, la televisión, las redes sociales, etc. Poco a poco, cuando la situación sanitaria lo fue permitiendo, se empezaron a planificar acciones de comunicación y relaciones públicas en las que se compaginaban los escenarios virtuales y una escasa presencialidad que debía respetar necesariamente la distancia social y las medidas sanitarias impuestas por cada gobierno. A medida que el proceso de vacunación avanzaba y los ingresos hospitalarios por Covid-19 disminuían, las herramientas de comunicación tradicionales empezaron a ganar terreno, aun siendo limitadas, y así se ejecutaron eventos familiares, empresariales, institucionales, organizacionales, deportivos... tanto a nivel local como internacional.

Los profesionales han tenido que improvisar, en todos los ámbitos, también los que trabajaban en publicidad o relaciones públicas; sin embargo, los sistemas educativos, la universidad, no ha aprovechado el aprendizaje para poner en valor la creatividad y la innovación en todos los estudios ofertados. El sistema educativo debe ser consciente de esta necesidad de formar a los estudiantes para adaptarse al entorno cambiante y salir airosamente de situaciones adversas.

Cierto es que nadie podía imaginar la magnitud de la pandemia, pero no menos cierto es que debemos aprender la lección y formar a los futuros profesionales, no sólo del campo de la comunicación, para que puedan utilizar herramientas creativas que les permitan hacer frente y minimizar las consecuencias de situaciones de crisis que, en mayor o menor magnitud, aparecerán casi a diario en sus vidas.

Cuando el sistema universitario europeo decidió unificar criterios en torno al conocido Plan Bolonia se pretendía actualizar sus métodos de enseñanza para atender a las demandas y realidades del siglo XXI y adecuar las enseñanzas a las necesidades del mercado laboral. Para ello (Esquivias, 2009) se reclamaba una en- 
señanza renovada con tintes creativos y con matices transdisciplinares. Pero en vista de los planes de estudio actuales, esta premisa no ha sido cumplida: la oferta transdisciplinar es escasa ya que los sistemas no permiten a los estudiantes diseñar su propio curriculum al estar inmersos en planes de estudio estáticos que sólo se adaptan a sus necesidades particulares a través de una reducida oferta de materias optativas, generalmente vinculadas a la propia titulación y, en contadas ocasiones, dentro de planes de estudios de otros ámbitos. Tampoco los tintes creativos se identifican con facilidad, ya que los grados y licenciaturas donde aparece nominalmente la creatividad son aquellos circunscritos al ámbito de la comunicación, por lo general, y al campo publicitario, en concreto.

Sin embargo, en lo que respecta a las publicaciones científicas y académicas sí se viene notando una mayor presencia de la creatividad, que muchas veces aparece vinculada con la innovación, las nuevas tecnologías y la gestión de crisis. Así, a nivel internacional el Ranking de revistas de Scimago (www.scopus.com) recoge a día de hoy 46 títulos que en su nomenclatura tiene la palabra "Creatividad" y el Journal Citation Reports, 5. No todas estas revistas están vinculadas al ámbito de la comunicación, sino también a áreas como la psicología, la educación, informática, diseño y artes o ingenierías.

Si los profesores que investigan enseñan mejor (Guzmán, 2018; Saavedra, 2017; Fernández-García CM, Rodríguez-Álvarez M \& Viñuela-Hernández, 2021) y cada vez existen más revistas especializadas en creatividad podríamos entender que los docentes dan peso a estos contenidos en sus materias, sin embargo, este hecho no se ve trasladado a los planes de estudio específicos $\mathrm{y}$, menos aún de forma transversal. Según De la Torre (2010) el profesor universitario es un profesional de la enseñanza superior innovadora y creativa, con dominio del contenido formativo y de estrategias didácticas capaz de hacer que los alumnos se entusiasmen por aprender y la enseñanza creativa se caracteriza por ser activa, motivadora y dinámica. Por ello, ya que contamos con docentes universitarios, especialistas en creatividad y sabedores de su importancia en la formación de los futuros profesionales, deben ser ellos los que presionen a las autoridades académicas a apostar por el cumplimiento de las bases del sistema europeo superior, de forma que los contenidos creativos se trabajen en la universidad de forma transversal e interdisciplinar y no solamente aplicadas a materias específicas. 
Si pretendemos orientar a los estudiantes a ser innovadores y a adaptarse a su entorno en todas las circunstancias, la creatividad es la base que puede permitirlo.

\section{De la formación en creatividad a los trabajos creativos}

La industria creativa abarca las actividades económicas basadas en el conocimiento e incluyen industrias como la publicidad, las artes, la arquitectura, la moda, el diseño, el cine, la fotografía, las artes escénicas, la música, las publicaciones, la televisión y la radio, el software... y es evidente que adquiere más peso cada día.

Si en 2018, en España, las industrias creativas empleaban a 5843000 personas, y suponían un 2'9€ del VAB total de España (Murciano, 2018) frente a los datos de Portugal, donde estas empresas eran el $2{ }^{\prime} \%$ del VAB del país y contaban con el $1^{\prime} 9 \%$ del total de empleos (Pereira, 2018) la situación a futuro promete, puesto que tal y como indica Isabelle Durant, Secretaria General Adjunta de la Conferencia de Naciones Unidas para el Comercio y el Desarrollo, “las industrias creativas son fundamentales para la agenda de desarrollo sostenible; estimulan la innovación y la diversificación de la economía y son un factor importante en el floreciente sector de servicios, apoyan el espíritu empresarial y contribuyen a la diversidad cultural". Tras casi un año de cuarentena por la pandemia, la ONU ha decidido dedicar este año 2021 a la economía creativa y ha declarado este año el Año Internacional de la Economía Creativa para el Desarrollo Sostenible (UNCTAD, 2021). A pesar de que todavía no se manejan datos estimaciones sobre el comercio mundial para la economía creativa en 2020, se espera que la recuperación económica empiece a notarse a medida que avance la vacunación y que las industrias creativas sean los motores de la economía mundial, ya que la tecnología, la digitalización y la conectividad, dominantes en la economía creativa dentro del segmento de software e I+D son fundamentales en un mundo post Covid-19.

En este marco, los gobiernos deben garantizar las infraestructuras para que las empresas creativas puedan desarrollarse y las instituciones académicas deben proveer de formación específica para que los empleados puedan hacer frente a los nuevos retos (Bakhshi \& Windsor, 2015). 


\section{Discusión y notas finales}

Se propone, brevemente, la discusión en torno a la triangulación Creatividad Retos Sociales - enseñanza de la Creatividad. La creatividad, concepto cada vez más relevante tanto en el mercado, como en las políticas públicas o en la academia, y puede ser reconocida como parte necesaria para abarcar la superación de los desafíos del siglo XXI, sobre todo, en un marco post-pandemia. Los diversos significados de la creatividad explorados al principio de este artículo nos permiten comprender el valor de la creatividad aplicada a diversos campos, incluyendo la academia y la ciencia. En este contexto, la relevancia que los gobiernos han atribuido a los expertos/científicos en la gestión de la crisis provocada por el Covid-19 - en la que se han apoyado en parte para la toma de decisiones - es un reconocimiento de este valor dado al concepto de la creatividad. A su vez, la respuesta que la comunidad científica consiguió darle es un reflejo de la creatividad orientada a la transferencia de conocimiento. Y es también una expresión del ejercicio de la creatividad coletiva (actividad que surge de la colaboración y aportación de muchos científicos) realizado en el descubrimiento de una vacuna, o en el análisis rápido del fenómeno de la pandemia a nivel de salud, pero también a nivel social y económico, análisis que se convierten en instrucciones y directrices para público profesional y ciudadano, incluso en el ámbito de la comunicación de crisis. Con respecto a la salud, la creatividad y la innovación se han expresado, por ejemplo, en soluciones como las destilerías de alcohol que han producido desinfectantes de manos, protectores faciales de impresión 3D o la creación de nuevas máquinas de ventilación. En definitiva, el uso de técnicas creativas de resolución de problemas se ha convertido en un aliado de la industria (Cohen \& Cromwell, 2021).

En cuanto al panorama de la enseñanza en las universidades en función del contexto de transformación de las sociedades en general y del mercado laboral en particular, el estudio presentado muestra que la presencia de la creatividad en los planes de estudio de las universidades españolas y portuguesas es residual y prácticamente se limita a las especialidades vinculadas con la comunicación. Lejos de jugar un papel preponderante en la formación de los universitarios para dotarles de herramientas que les permitan desarrollarse profesionalmente en un entorno cambiante, el sistema de educación superior sigue viviendo de espaldas 
a las necesidades profesionales y no forma a sus estudiantes con contenidos que les permitan desarrollar su pensamiento divergente para encontrar soluciones a problemas y adaptarse con mayor facilidad a los entornos cambiantes. En este sentido, podríamos abogar por la creación de materias específicas de creatividad que se incluyesen en los planes de estudio de manera concreta para las distintas especialidades, pero también de forma transversal; así, los estudiantes tendrían la oportunidad de trabajar estos contenidos y desarrollarlos de cara a un futuro laboral en el que el cambio es una constante.

A pesar de ello, consideramos que tanto el incremento de publicaciones científicas y académicas reflexionando sobre el tema, como la presión de las entidades supranacionales y los mercados laborales propios de cada país, junto con la experiencia de adaptación al cambio impuesta por la Covid-19, harán que los sistemas de enseñanza superior tengan que asumir el peso que la creatividad debe jugar en la formación de sus estudiantes.

En resumen, "el objetivo principal de la educación es crear personas capaces de hacer cosas, no simplemente repetir lo que otras generaciones han hecho - personas creativas, inventivas e investigadoras" (Ginsberg \& Opper, 1969, p. 5, cit in Hadzigeorgiou et. $\mathrm{Al}, 2012$ ). En este orden de ideas, la creatividad es un recurso increíble, contínuo y en constante transformación (Balonas, 2020).

\section{Referencias}

Albert, R. S., \& Runco, M. A. (1999). A History of Research on Creativity. In Sternberg, R.J. (Ed.). Handbook of Creativity. United Kingdom: Cambridge University Press.

Balonas, S. (2019). “Que a Força esteja contigo - os desafios da publicidade na nova galáxia comunicacional", in Ferreira, I. (ed.) Publicidade: teorias, métodos e práticas | Advertising: theories, methods and practices. Media \& Jornalismo Revista do Centro de Investigação Media e Jornalismo. Volume $\mathrm{N}^{\circ}$ 34. (pp.1334). https://impactum-journals.uc.pt/mj/issue/view/2183-5462 34

Balonas, S. (2020). "Crise e transformação - um ensaio sobre os catalisadores da mudança", in Oliveira, M., Machado, H., Sarmento, J. \& Ribeiro, M. (coord). 
La creatividad en la enseñanza como factor de aproximación de la universidad... | 33

MONOGRÁFICO

Sociedade e crise(s). Braga: UMinho Editora. D0I https://doi.org/10.21814/ uminho.ed.21

Bakhshi, H., Windsor, G. (2015). The creative economy and the future of employment. London: Nesta.

Beriain A. \& Gascón J.F.F. (2013). Double-dregree university graduates: a case study in Spain. International Journal of Interdisciplinary Educational Studies, 2013, vol. 7, num. 1, p. 87-96.

Boorstin, D. J. (1993). Os Criadores. Uma História dos Heróis da Imaginação. Lisboa: Gradiva Publicações.

Castillo Esparcia, A., (2013). “EEES y la formación de comunicación en España. La estructura de la oferta de posgrado". Estudios del mensaje periodístico, 19, 99-110. Cohen, A., Cromwell, J. (2021). How to Respond to the COVID-19 Pandemic with More Creativity and Innovation. Population Health Management. Vol. 24, N. 2, Mary Ann Liebert, Inc. DOI: 10.1089/pop.2020.0119, consultado el 23 mayo 2021.

Cuenca-Fontbona J., Compte-Pujol, M., Matilla K.,Hernández-Martínez, S., (2017). Un análisis de los masters universitarios especializados en Relaciones Públicas y comunicación corporativa (curso 2016-17) desde la perspectiva de las Relaciones Públicas, Obra Digital, (13), 37-57. Httpps:// doi.org/10.25029/ od.2017.156.13.

Damásio, A. (1994). 0 Erro de Descartes. Emoção, Razão e Cérebro Humano. MemMartins: Publicações Europa-América.

David, A. P. \& Morais, M. d. F. (2012). Pensando a Criatividade: apontamentos sobre o percurso explicativo do conceito. Revista Recrearte: p. 16. Consultado en: https://repositorium.sdum.uminho.pt/bitstream/1822/20475/1/revista\%20 Recrearte\%20.pdf

De la Torre, S. (2010). La universidad que queremos. Estrategias creativas en el aula universitaria. Revista Digital Universitaria, Vol, 10, num. 12. http://www. revista.unam.mx/vol.10/num12/art85/int85.htm

De Masi, D. (2002). Criatividade e Grupos Criativos. Fantasia e Concretude. Vol.2. Editora Sextante.

Esquivias (2009). “Enseñanza creativa y transdisciplinar para una nueva universidad. Encuentros multidisciplinares, 43. 
Fanjul Peyró, C; Pérez Serrano, M.J; Cabezuelo Lorenzo, F. (2010). La guía docente como herramienta activa en la praxis didáctica de materias de Publicidad y Relaciones Públicas adaptadas al EEES, @tic. Revista de innovación educativa, $\mathrm{N}^{0}$ 4, enero-junio, 2010, pp 81-84.

Florida, R. (2013). Building the creative economy: an interview with Richard Florida. December. McKinsey \& Company.

Fontes, H. (27 octubre 2020). Desafios das PME portuguesas na economia pósCOVID. AETICE. Consultado el 22 de enero de 2021. https://www.aetice. $\mathrm{pt} / 2020 / 10 / 27 /$ desafios-pme-portuguesas/

Gardner, H. (2010). Five minds for the future. Cambridge, MA: Harvard Business School Press.

Guzmán, J. C. (2018). Las buenas prácticas de enseñanza de los profesores de educación superior. REICE. Revista Iberoamericana sobre Calidad, Eficacia y Cambio en Educación.

Fernández-García, C. M., Rodríguez-Álvarez, M., \& Viñuela-Hernández, M. P. (2021). La percepción de los estudiantes universitarios acerca de la eficacia docente. Efectos sobre el compromiso de los estudiantes. Revista de Psicodidáctica, 26(1), 62-69.

Hadzigeorgiou, Y., Fokialis, P., Kabouropoulou, M. (2012). Thinking about Creativity in Science Education. Creative Education. Vol.3, No.5, 603-611 consultado en: https://www.scirp.org/html/22940.html

Hollanders, H. \& Cruysen, A. v. (2009). Design, Creativity and Innovation: a Scoreboard Approach. UNU- MERIT, Maastrich University, Maastrich, the Netherlands.

Lund, S., Madgavkar, A, Manyika, J., Smit, S., Ellingrud, K., Meany, M, Robinson, 0. (2021). The future of work after COVID-19. McKinsey Global Institute

Kaufman, J. C. \& Beghetto, R. A. (2009). Beyond Big and Little: The Four C Model of Creativity. Review of General Psychology, 13(1), 1-12. http://dx.doi. org/10.1037/a0013688.

May, R. (1975). The Courage to Create. New York: W.W. Norton \& Co.

Ossiannilsson E. (2021), Some Challenges for Universities, in a Post Crisis, as Covid-19. In: Burgos D., Tlili A., Tabacco A. (eds) Radical Solutions for Education in a Crisis Context. Lecture Notes in Educational Technology. Springer, Singapore. https://doi.org/10.1007/978-981-15-7869-4 7 Consultado en a https://link. springer.com/chapter/10.1007/978-981-15-7869-4 7) 
Pink, D. (2005). A Whole New Mind. Australia: Allen\&Unwin, 1.

Rodríguez, I., (2009). La Enseñanza de la Creatividad en los estudios de Publicidad de las Universidades Españolas. Tesina.

Saavedra, Y. R. (2017). De una universidad que enseña a una universidad que investiga. Revista Gestión y Finanzas, 1(2).

Silva, N. (15 diciembre 2020). Estudo conclui que 70\% das empresas pretende adotar um sistema misto de trabalho após a pandemia. SAPO. Consultado el 22 enero de 2021 https://jornaleconomico.sapo.pt/noticias/estudo-concluique-70-das-empresas-pretende-adotar-um-sistema-misto-de-trabalho-apos-apandemia-677046

Stenfors, S, Tanner, L. (2006). High-level decision support in companies: where is the support for creativity and innovation? In Adam F., Brezillon, P., Carlsson, S., \& Humphreys P. (eds). Creativity and Innovation in Decision Making and Decision Support. Vol.1, Ludic London.

UNCTAD (2021). La economía creativa tendrá su momento en el año 2021. Consltado en https://unctad.org/es/news/la-economia-creativa-tendra-su-momentoen-el-ano-2021

UNESCO. (2020). Futures of education. Consultado en https://en.unesco.org/ futuresofeducation/

Wallas, G. (1926). The Art of Thought. New York: Harcourt Brace.

\section{(c)}

Este obra está bajo una licencia de Creative Commons Reconocimiento 4.0 Internacional. 